arXiv: accepted by Phys. Rev. Lett.

\title{
Explosive Turbulent Magnetic Reconnection
}

\author{
K. Higashimori, ${ }^{1}$ N. Yokoi ${ }^{2}$ and M. Hoshino ${ }^{1}$ \\ ${ }^{1}$ Department of Earth and Planetary Science, University of Tokyo \\ ${ }^{2}$ Institute of Industrial Science, University of Tokyo
}

(Dated: March 9, 2022)

\begin{abstract}
We report simulation results for turbulent magnetic reconnection obtained using a newly developed Reynolds-averaged magnetohydrodynamics model. We find that the initial Harris current sheet develops in three ways, depending on the strength of turbulence: laminar reconnection, turbulent reconnection, and turbulent diffusion. The turbulent reconnection explosively converts the magnetic field energy into both kinetic and thermal energy of plasmas, and generates open fast reconnection jets. This fast turbulent reconnection is achieved by the localization of turbulent diffusion. Additionally, localized structure forms through the interaction of the mean field and turbulence.
\end{abstract}

Critical questions relating to magnetic reconnection are how and when does fast reconnection take place, particularly in the case of a high magnetic Reynolds number $\left(R_{m} \sim 10^{10}\right)$. Because a magnetic Reynolds number is sufficiently high to maintain turbulence in space, much attention has been paid to the relationship between turbulence and magnetic reconnection [1]. For example, it has been theoretically suggested that in strong Alfvénic turbulence, the reconnection rate does not depend on electric resistivity, but rather on the properties of turbulence (such as the characteristic scale length and power of the fluctuation) [2]. The scenario has been examined in magnetohydrodynamics (MHD) simulations, where external turbulence is forced in a current sheet [3]. The effect of turbulence on the reconnection rate has also been investigated in large-scale two-dimensional simulation; it was suggested that there is a critical turbulent power above which turbulence greatly affects reconnection and the reconnection rate has weaker dependence on electric resistivity than does Sweet-Parker [4, 5] reconnection [6]. Another study focused on reconnection in turbulence in terms of a turbulent cascade, and it was suggested that reconnection rates are distributed and controlled by turbulence [7]. Recently, another viewpoint on the relationship between turbulence and reconnection has been presented according to the theory of MHD turbulence [8]. In that study, it was theoretically suggested that the generation of cross-helicity $\mathcal{W} \equiv\left\langle\boldsymbol{v}^{\prime} \cdot \boldsymbol{b}^{\prime}\right\rangle$ (where $\boldsymbol{v}^{\prime}$ and $\boldsymbol{b}^{\prime}$ are respectively the characteristic velocity and magnetic field of the turbulent motion) dramatically enhance the rate of reconnection.

We apply a Reynolds-averaged MHD model and investigate the nonlinear evolution of turbulent reconnection. In the model, physical quantities (such as the velocity $\boldsymbol{v}$ ) are decomposed into mean and turbulent quantities; e.g., $\boldsymbol{v}=\boldsymbol{V}+\boldsymbol{v}^{\prime}$ (where the capital letter stands for the mean quantity). In this study, tubulent effects are included in Ohm's law. Taking the ensemble average, $\langle\ldots\rangle$ (e.g., $\langle\boldsymbol{v}\rangle=\boldsymbol{V}$ ), of the Ohm's law gives a following equation:

$$
\boldsymbol{E}=\eta \boldsymbol{J}-\boldsymbol{V} \times \boldsymbol{B}-\left\langle\boldsymbol{v}^{\prime} \times \boldsymbol{b}^{\prime}\right\rangle,
$$

where $\left\langle\boldsymbol{v}^{\prime} \times \boldsymbol{b}^{\prime}\right\rangle$ is the electromotive force due to effects of turbulence, and it modulates the mean electric field. Mean variables, such as $\boldsymbol{V}$ and $\boldsymbol{B}$, are obtained using ordinal MHD equations:

$$
\begin{aligned}
& \frac{\partial \rho}{\partial t}+\nabla \cdot(\rho \boldsymbol{V})=0,(2) \\
& \frac{\partial}{\partial t}(\rho \boldsymbol{V})+\boldsymbol{\nabla} \cdot\left[\rho \boldsymbol{V} \boldsymbol{V}+\left(P+\frac{B^{2}}{2}\right) \mathbf{I}-\boldsymbol{B} \boldsymbol{B}\right]=\mathbf{0},(3) \\
& \frac{\partial}{\partial t}\left(\frac{P}{\gamma_{a}-1}+\frac{\rho}{2} V^{2}+\frac{B^{2}}{2}\right) \\
& +\boldsymbol{\nabla} \cdot\left[\left(\frac{\gamma_{a}}{\gamma_{a}-1} P+\frac{\rho}{2} V^{2}\right) \boldsymbol{V}+\boldsymbol{E} \times \boldsymbol{B}\right]=0,(4) \\
& \frac{\partial \boldsymbol{B}}{\partial t}+\boldsymbol{\nabla} \times \boldsymbol{E}=\mathbf{0},(5)
\end{aligned}
$$

where $\mathbf{I}$ is the unit tensor. Note that the velocity is normalized by the Alfvén velocity. $\gamma_{a}$ is an adiabatic index and set to $\gamma_{a}=5 / 3$.

The most important part of modeling turbulence is the evaluation of the electromotive force, $\left\langle\boldsymbol{v}^{\prime} \times \boldsymbol{b}^{\prime}\right\rangle$, which reflects the information of turbulence. Here, we applied the model for inhomogeneous MHD turbulence [9 11]. In this model, the electromotive force can be written as

$$
\left\langle\boldsymbol{v}^{\prime} \times \boldsymbol{b}^{\prime}\right\rangle=-\beta_{t} \boldsymbol{J}+\gamma_{t} \boldsymbol{\Omega},
$$

where $\boldsymbol{\Omega}=\boldsymbol{\nabla} \times \boldsymbol{V}$ is the mean vorticity. $\beta_{t}$ and $\gamma_{t}$ are respectively expressed as $\beta_{t}=C_{\beta} \tau \mathcal{K}$ and $\gamma_{t}=C_{\gamma} \tau \mathcal{W}$. $\mathcal{K} \equiv\left\langle v^{\prime 2}+b^{\prime 2}\right\rangle / 2$ and $\mathcal{W}=\left\langle\boldsymbol{v}^{\prime} \cdot \boldsymbol{b}^{\prime}\right\rangle$ are respectively the macroscopically defined turbulent energy and crosshelicity. $\tau$ is the characteristic time scale of turbulence. $C_{\beta}$ and $C_{\gamma}$ are model constants of $O\left(10^{-1}\right)$. (We checked that the result did not strongly depend on their exact values.) We set $C_{\beta}=C_{\gamma}=0.3$ in the present paper. The advantage of this model is that the coefficients $\beta_{t}$ and $\gamma_{t}$ are determined by the following equations for turbulence [12]:

$$
\begin{gathered}
\frac{\partial \mathcal{K}}{\partial t}=-\left\langle\boldsymbol{v}^{\prime} \times \boldsymbol{b}^{\prime}\right\rangle \cdot \boldsymbol{J}+\boldsymbol{B} \cdot \nabla \mathcal{W}-\boldsymbol{V} \cdot \nabla \mathcal{K}-\epsilon_{\mathcal{K}}, \\
\frac{\partial \mathcal{W}}{\partial t}=-\left\langle\boldsymbol{v}^{\prime} \times \boldsymbol{b}^{\prime}\right\rangle \cdot \boldsymbol{\Omega}+\boldsymbol{B} \cdot \nabla \mathcal{K}-\boldsymbol{V} \cdot \boldsymbol{\nabla} \mathcal{W}-\epsilon_{\mathcal{W}},
\end{gathered}
$$


where $\epsilon_{\mathcal{K}}$ and $\epsilon_{\mathcal{W}}$ are respectively the dissipation rates of turbulence energy and cross-helicity. They are given by

$$
\begin{gathered}
\epsilon_{\mathcal{K}}=\frac{\mathcal{K}}{\tau}, \\
\epsilon_{\mathcal{W}}=C_{\mathcal{W}} \frac{\mathcal{W}}{\tau},
\end{gathered}
$$

where $C_{\mathcal{W}}$ is a model constant [13], and set to 1.3 here. The above set of equations (11)-(10) is numerically solved using the two-dimensional ( $x-z$ plane) fourthorder Runge-Kutta and fourth-order central difference scheme 14]. Grid intervals $\Delta x$ and $\Delta z$ are fixed to unity, and the simulation size is $L_{x} \times L_{z}=2048 \times 512$. (The simulation box ranges $|x| / L_{z} \leq 2.0$ and $-0.25 \leq z / L_{z} \leq$ 0.75.) Boundaries in $x$ - and $z$-directions are both periodic, and a pair of Harris current sheets is assumed. Hereafter, only the region $|z| / L_{z} \leq 0.25$ (i.e., the lower current sheet) is discussed.

In this simulation model, we must give the initial conditions for both the mean field (such as $\boldsymbol{B}$ and $P$ ) and the turbulent field (such as $\mathcal{K}$ and $\mathcal{W}$ ). As for the initial mean field, the mean magnetic field for the lower current sheet $\left(|z| / L_{z} \leq 0.25\right)$ is given by $\boldsymbol{B}=$ $B_{x 0} \tanh (z / \delta) \boldsymbol{e}_{x}+B_{z 0} \sum_{m=1}^{10} \sin \left(2 \pi m x / L_{x}\right) \boldsymbol{e}_{z}$, where $B_{x 0}=1.0$ and $B_{z 0} / B_{x 0}=1.0 \times 10^{-3} . \delta\left(=0.02 L_{z}\right)$ is half-thickness of the initial current sheets. $\boldsymbol{e}_{x}$ and $\boldsymbol{e}_{z}$ are respectively the unit vectors in $x$ - and $z$-directions. The plasma beta outside the current sheets is set to $\beta_{p}=0.5$, and the spatial distribution of $P$ is determined to satisfy the pressure balance. Additionally, uniform electric resistivity, $\eta=1.0 \times 10^{-2}$, is assumed to avoid numerical instability.

As for the initial turbulent field, we assume $\mathcal{W}=0$ and $\mathcal{K}=1.0 \times 10^{-2}$ everywhere. We found that the magnitude of $\mathcal{K}$ did not change the basic property of turbulent reconnection. In addition, a steady state of turbulent energy, $\partial \mathcal{K} / \partial t=0$, is assumed in the initial current sheets. Because $\boldsymbol{\Omega}=\boldsymbol{V}=\mathbf{0}$ and $\mathcal{W}=0$ at the center of the current sheets, it holds that $\tau_{0}=C_{\beta}^{-1 / 2}|\boldsymbol{J}|_{z=0}^{-1}$ in the steady state. (Here equations (6), (7), and (9) are used.) To investigate the relationship between turbulence and reconnection, we slide $\tau$ from the steady state, $\tau=\tau_{0}$, by introducing the parameter $C_{\tau}$ as $\tau=C_{\tau} \tau_{0}$. It should be noted that $\tau$ is constant throughout each simulation run. We execute simulations with different $C_{\tau}$ values, and the characteristic cases $\mathrm{A}-\mathrm{D}$ are summarized in Table I. (The total number of simulation runs corresponds to the number of red points in Figure 2 ) Since $C_{\tau}$ determines the characteristic time scale of turbulence, it controls dissipation rate of turbulent energy, $\epsilon_{\mathcal{K}}$. For example, if $C_{\tau}$ is much smaller than unity, $\epsilon_{\mathcal{K}}$ becomes $\epsilon_{\mathcal{K}} \gg 1$, and $\mathcal{K} \rightarrow 0$ is expected as the simulation time passes. In such a case, the system will develop into laminar flow. On the other hand, in the case of $C_{\tau} \gg 1$, it is expected that the system quickly becomes turbulent, and reconnection will not occur owing to strong turbulent diffusion. (Here it is noted from equations (11), (5), and (6) that the $\beta_{t}$-related term in the induction equation is
TABLE I. Simulation parameters

\begin{tabular}{cccccccc}
\hline \hline Run & $\mathrm{A}$ & $\cdots$ & $\mathrm{B}$ & $\cdots$ & $\mathrm{C}$ & $\cdots$ & $\mathrm{D}$ \\
\hline$C_{\tau}$ & 0.05 & $\cdots$ & 0.5 & $\cdots$ & 1.2 & $\cdots$ & 3.0 \\
\hline \hline
\end{tabular}

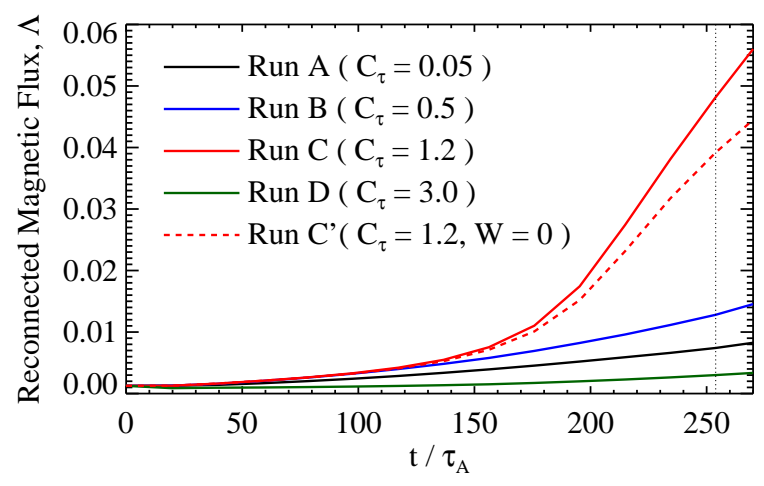

FIG. 1. Time evolution of the reconnected magnetic fluxes in the cases of $C_{\tau}=0.05$ (black), $C_{\tau}=0.5$ (blue), $C_{\tau}=1.2$ (red), and $C_{\tau}=3.0$ (dark green). In Run (C') (red-dashed), $\mathcal{W}$ is forced to be 0 throughout the calculation.

$\partial \boldsymbol{B} / \partial t=\cdots-\boldsymbol{\nabla} \times\left[\left(\eta+\beta_{t}\right) \boldsymbol{J}\right]$, and the turbulent diffusion of the mean magnetic field increases as $\mathcal{K}$ increases.) Therefore, in this study, we focus on the most interesting parameters around $C_{\tau} \sim 1$.

Figure 1 shows the time evolution of the reconnected magnetic flux, $\Lambda \equiv \int_{-L_{x} / 2}^{+L_{x} / 2} d x\left|B_{z}\right|_{z=0} /\left(B_{x 0} L_{x}\right)$, for simulation runs (A)-(D). (In Run (C'), the cross helicity, $\mathcal{W}$, is switched off in order to discuss the contribution of $\mathcal{W}$ to the reconnection rate. This is referred to later.) Time is normalized by the Alfvén transit time, $\tau_{A} \equiv \delta / V_{A}$. In the present paper, we do not discuss the evolution at time $t / \tau_{A}>260$ to avoid the boundary effect on the reconnection [15]. The black line shows the reconnected flux, $\Lambda$, in the case of $C_{\tau}=0.05$. In this case, both $\beta_{t}$ and $\gamma_{t}$ soon

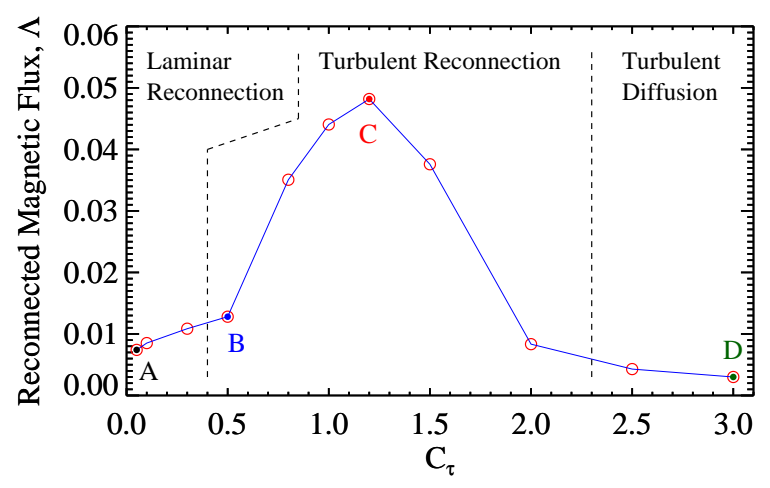

FIG. 2. Reconnected magnetic fluxes at $t / \tau_{A}=254$ vs $C_{\tau}$. Red points stand for simulation runs with different $C_{\tau}$ values. 


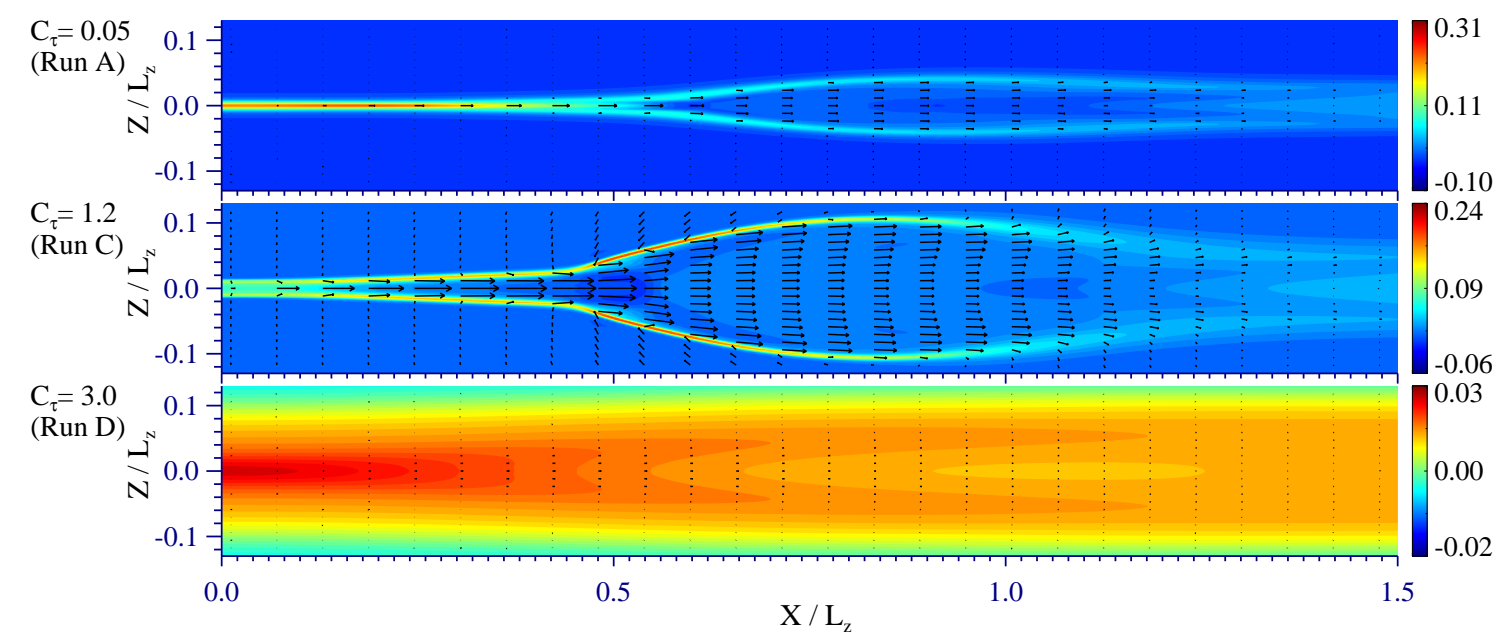

FIG. 3. $y$ components of the electric currents in the laminar $\left(C_{\tau}=0.05\right)$ and turbulent $\left(C_{\tau}=1.2,3.0\right)$ cases at $t / \tau_{A}=254$ are shown as color contour plots. Black arrows show the flow velocity.

drop to zero, and the reconnection becomes slow laminar one. As $C_{\tau}$ increases, reconnection develops more quickly, and the development is fastest around $C_{\tau} \sim 1.2$ (see the red line) for the present simulation parameters. However, with larger $C_{\tau}$, the speed of reconnection again drops (see the dark-green line), and falls below that of laminar reconnection. In this case, the turbulent energy $\mathcal{K}$ quickly increases, and the resultant strong turbulent diffusion in the initial current sheet prevents reconnection. As a result, no outflow jet is observed. (The profile of the velocity is shown in the bottom panel of Figure 3 as black arrows.) Figure 2 shows the relationship between reconnected magnetic fluxes and the parameter $C_{\tau}$ at $t / \tau_{A}=254$ for all simulation runs. The figure clearly shows that the initial current sheet develops in three ways depending on $C_{\tau}$ values and the speed of reconnection is fastest for a moderate $C_{\tau}$ value $\left(C_{\tau} \sim 1\right)$.

We now discuss the spatial structures of the above three cases. In Figure 3, the $y$ components of the electric current at $t / \tau_{A}=254$ in the laminar $\left(C_{\tau}=0.05\right)$ and turbulent $\left(C_{\tau}=1.2,3.0\right)$ cases are shown as contour plots. Flow vectors are overlaid as black arrows. In the laminar case, a Sweet-Parker-type current sheet forms [16, 17], and reconnection is more gradual than in the turbulent case. Under strong turbulence $\left(C_{\tau}=3.0\right)$, the initial current sheet (with thickness $\sim 0.04 z / L_{z}$ ) quickly broadens and reconnection does not take place, as shown in the bottom panel. On the other hand, in the case of turbulent reconnection $\left(C_{\tau}=1.2\right.$ : see the middle panel), two pairs of current sheets formed as Petschek-type reconnection [18], and open reconnection jets are observed. The existence of Petschek-type reconnection itself has already supported by the results of anomalous resistivity models [19, 20], however, the inherent physical processes of turbulent reconnection are quite different from them. It should manifest that the physics is self-consistently determined by the nature of MHD turbulence.

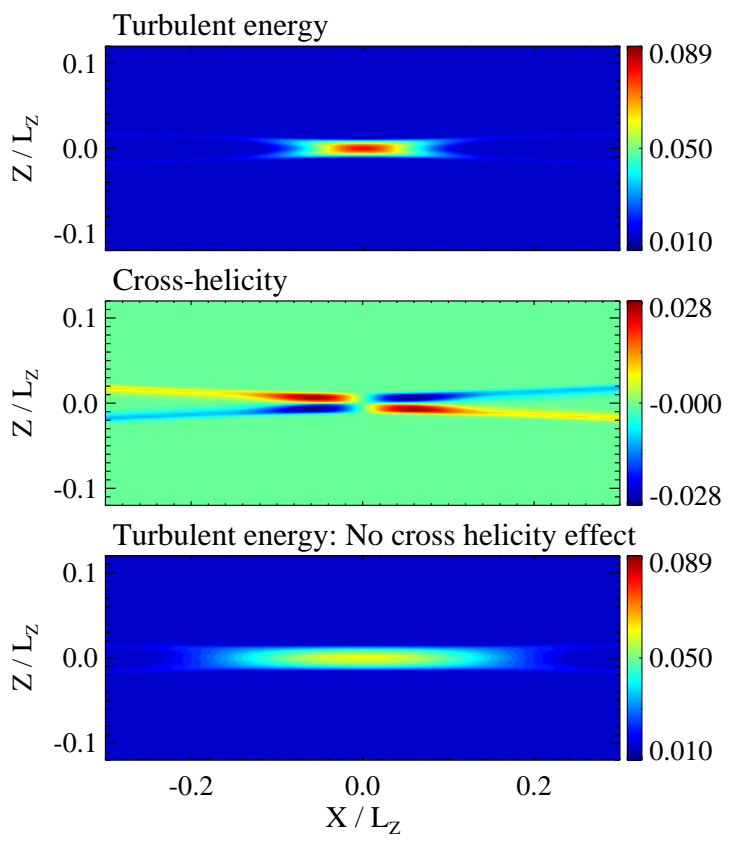

FIG. 4. The upper two contour plots show the spatial distributions of the turbulent energy $\mathcal{K}$ and the cross-helicity $\mathcal{W}$ near the magnetic neutral point in the case of $C_{\tau}=1.2$. The bottom panel shows the spatial distribution of $\mathcal{K}$ at $C_{\tau}=1.2$, where $\mathcal{W}$ is switched off throughout the calculation. These three snapshots are taken at time $t / \tau_{A}=254$.

In such fast reconnection cases, both the turbulent energy $\mathcal{K}$ and cross-helicity $\mathcal{W}$ are efficiently produced, and the turbulent diffusion near the magnetic neutral point is locally strengthened. The upper two panels of Figure 4 show the spatial distributions of the turbulent energy $\mathcal{K}$ and cross-helicity $\mathcal{W}$ near the turbulent diffusion region at time $t / \tau_{A}=254$ in the case of $C_{\tau}=1.2$. These spatial distributions of $\mathcal{K}$ and $\mathcal{W}$ are 
determined by the interaction between the mean and turbulent fields. In the first stage of turbulent reconnection, as $\boldsymbol{V} \sim \mathbf{0}$ and $\mathcal{W} \sim 0$, the production term of equation (77), $-\left\langle\boldsymbol{v}^{\prime} \times \boldsymbol{b}^{\prime}\right\rangle \cdot \boldsymbol{J} \sim \beta_{t} \boldsymbol{J}^{2}$, dominates. The increasing $\beta_{t}=C_{\beta} \tau \mathcal{K}$ then facilitates turbulent diffusion and triggers reconnection. Subsequently, topological change in the magnetic field and vorticity develops. The electric current and vorticity generate the cross-helicity, according to the production term of equation (8); i.e., $-\left\langle\boldsymbol{v}^{\prime} \times \boldsymbol{b}^{\prime}\right\rangle \cdot \boldsymbol{\Omega}=\beta_{t} \boldsymbol{J} \cdot \boldsymbol{\Omega}-\gamma_{t} \boldsymbol{\Omega}^{2}$. In this way, a quadrupole structure of $\mathcal{W}$ forms. Then, $\mathcal{W}$ plays an important role on the development of the localized turbulent diffusion region. In the absence of $\mathcal{W}$, the turbulent diffusion region broadens as is shown in the bottom panel of Figure 4. On the other hand in the presence of $\mathcal{W}, \mathcal{K}$ is locally strengthened and the reconnection rate increases compared to that in the $\mathcal{W}=0$ case (see red-solid and red-dashed lines in Figure 1).

In this paper, the relation between turbulence and magnetic reconnection was investigated using the Reynolds-averaged MHD model, where mean and turbulent fields develop by interacting with each other. It was found that the initial current sheet develops in three ways: laminar reconnection, turbulent reconnection, and turbulent diffusion. Reconnection develops most quickly in the second case, and it macroscopically appears as a single X-type reconnection with open fast outflow jets owing to the locally strengthened turbulent diffusion. Such fast turbulent reconnection would, for example, play an important role in the region where there is a large gap between the overall and dissipation scales. It is not clear whether reconnection around the dissipation scale really develops into a huge-scale phenomenon. Assuming the existence of turbulence, however, the thickness of the current sheet does not necessarily become as thin as the dissipation scale, if $\beta_{t}>\eta$. Instead of dissipationscale physics, turbulent diffusion could macroscopically change the topology of (mean) magnetic fields. On the other hand, in the case that the scale gap is comparatively small, kinetic effects should be taken into account. From the viewpoint of turbulent reconnection, the outflow of collisionless magnetic reconnection could become turbulent without any forced perturbation 21. Additionally, along the boundary between inflow and outflow regions, Alfvén waves could be driven by the ion beam accelerated around the diffusion region 22]. We suggest that such self-generated turbulence and waves would macroscopically appear as sources of $\mathcal{K}$ and $\mathcal{W}$, and may further enhance the turbulent diffusion and the resultant reconnection rate.

The model allows us to investigate phenomena of MHD turbulence even in the case of a high magnetic Reynolds number, and we hope that this will contribute to studies where direct numerical simulation (DNS) is difficult or impossible. On the other hand, it should be mentioned that the model provides less accuracy than DNS. For example, in the present paper, the characteristic turbulent timescale $\tau$ is assumed to be constant, and this may result in overestimation of turbulent diffusion in the case of $C_{\tau} \gg 1$. The timescale of turbulence, as well as the dissipation rate of turbulent energy, should be determined according to the nonlinear dynamics of turbulence. In future works, the accuracy of the model needs to be improved through mutual understanding with DNS and observations to clarify the role of turbulence in various phenomena.

This research is supported by Japan Society for the Promotion of Science (JSPS; Grant No. 12J10000, 22001, and 24540228), and partially supported by NAOJ and NORDITA.
[1] W. H. Matthaeus and Space Sci. Rev. 160, 145 (2011) Astrophys. J. 517, 700 (1999)

[3] G. Kowal, A. Lazarian, E. T. Vishniac, and K. Otmianowska-Mazur, Astrophys. J. 700, 63 (2009).

[4] P. A. Sweet, in Electromagnetic Phenomena in Cosmical Physics, IAU Symposium, Vol. 6, edited by B. Lehnert (1958) p. 123.

[5] E. N. Parker, J. Geophys. Res. 62, 509 (1957)

[6] N. F. Loureiro, D. A. Uzdensky, A. A. Schekochihin, S. C. Cowley, and T. A. Yousef, Mon. Not. R. Astron. Soc. 399, L146 (2009).

[7] S. Servidio, W. H. Matthaeus, M. A. Shay, P. A. Cassak, and P. Dmitruk, Physical Review Letters 102, 115003 (2009).

[8] N. Yokoi and M. Hoshino, Physics of Plasmas 18, 111208 (2011).

[9] A. Yoshizawa, Physics of Fluids B 2, 1589 (1990)

[10] A. Yoshizawa and N. Yokoi, Astrophys. J. 407, 540 (1993)

[11] N. Yokoi, Geophys. Astrophys. Fluid Dyn. 107, Issue 1.
[12] N. Yokoi, R. Rubinstein, A. Yoshizawa, and F. Hamba, Journal of Turbulence 9, N37 (2008)

[13] N. Yokoi, Journal of Turbulence 12, N27 (2011).

[14] M. Rempel, M. Schüssler, and M. Knölker, Astrophys. J. 691, 640 (2009).

[15] T. G. Forbes and E. R. Priest, Reviews of Geophysics 25, 1583 (1987).

[16] D. Biskamp, Physics of Fluids 29, 1520 (1986)

[17] D. A. Uzdensky and R. M. Kulsrud, Physics of Plasmas 7, 4018 (2000)

[18] H. E. Petschek, NASA Special Publication 50, 425 (1964).

[19] T. Sato and T. Hayashi, Physics of Fluids 22, 1189 (1979).

[20] M. Ugai, Physics of Fluids B 4, 2953 (1992).

[21] W. Daughton, V. Roytershteyn, H. Karimabadi, L. Yin, B. J. Albright, B. Bergen, and K. J. Bowers, Nature Physics 7, 539 (2011).

[22] K. Higashimori and M. Hoshino, Journal of Geophysical Research 117, A01220 (2012) 